Artículo original

\title{
Incidencia y subregistro de casos de leptospirosis diagnosticados con tres métodos diferentes en Urabá, Colombia
}

\author{
Janeth Pérez-García ${ }^{1}$, Piedad Agudelo-Flórez¹, Gabriel Jaime Parra-Henao ${ }^{1}$, Jesús \\ Ernesto Ochoa ${ }^{2}$, Margarita Arboleda ${ }^{3}$ \\ ${ }^{1}$ Grupo de Epidemiologia y Bioestadística, Escuela de Graduados, Universidad CES, Medellín, \\ Colombia \\ ${ }^{2}$ Grupo de Epidemiología, Seminario Taller de Epidemiología Teórica, Facultad Nacional de \\ Salud Pública, Universidad de Antioquia, Medellín, Colombia \\ ${ }^{3}$ Grupo de Medicina Tropical, Instituto Colombiano de Medicina Tropical, Apartadó, Colombia
}

Introducción. La leptospirosis representa un problema de salud pública y es una causa importante de morbimortalidad en la región de Urabá, cuya notificación se ve afectada por las deficiencias en el diagnóstico.

Objetivo. Establecer la incidencia de la leptospirosis en los municipios del llamado 'eje bananero' de la región de Urabá, documentar la magnitud del subregistro y proponer orientaciones para el diagnóstico por laboratorio por parte de la red de salud pública. Materiales y métodos. Se compararon dos fuentes de información sobre la leptospirosis: el sistema oficial nacional de vigilancia y un estudio transversal de 479 pacientes febriles, llevado a cabo entre abril de 2010 y mayo de 2012. El diagnóstico se hizo con base en tres pruebas: inmunofluorescencia indirecta, microaglutinación y hemocultivo. La exhaustividad de cada fuente de información se estimó mediante el método de captura y recaptura. Resultados. El $58 \%$ (278/479) de los pacientes fueron positivos para leptospirosis, por lo menos, en una de las pruebas y, el 10,43\% (29/278), en las tres. La inclusión de una cepa nativa en el panel de la prueba de microaglutinación aumentó el porcentaje de positividad en $15 \%$. La tasa acumulada de incidencia fue de 66,5 por 100.000 habitantes y la proporción de letalidad fue de $2,15 \%$. El subregistro de la morbilidad por leptospirosis en la región de Urabá, fue de $27,8 \%$ y, el de la mortalidad, de $66,6 \%$.

Recibido: $20 / 06 / 18$

Aceptado: 25/10/18

Publicado: $07 / 11 / 18$

Citación:

Pérez-García J, Agudelo-Flórez P, Parra-Henao GJ, Ochoa JE, Arboleda M. Incidencia y subregistro de casos de leptospirosis diagnosticados con tres métodos diferentes en Urabá, Colombia. Biomédica. 2019;39(Supl.1):150-62

https://doi.org/10.7705/biomedica.v39i0.4577

\section{Correspondencia}

Margarita Arboleda, Carrera 98 № 106-176, Barrio Chinita, Apartadó, Colombia

Teléfonos: (574) 8281708 y (574) 8285606 marboleda@ces.edu.co y margaritarboleda@gmail. com

Contribución de los autores:

Janeth Pérez-García: análisis estadístico e interpretación de los datos

Piedad Agudelo-Flórez y Margarita Arboleda: concepción del estudio, obtención de la financiación y recolección de los datos

Gabriel Jaime Parra-Henao: concepción del estudio y revisión crítica

Jesús Ernesto Ochoa: concepción del estudio e interpretación de los datos

Todos los autores participaron en la redacción del manuscrito.

\section{Financiación:}

Proyecto financiado por Colciencias (código 122865740423) como parte del proyecto marco con código $325649326207-678$.

Conflicto de intereses:

Los autores declaran no tener ningún conflicto de intereses.
Conclusión. El subregistro de leptospirosis en la región reitera la necesidad de usar más de una prueba diagnóstica para identificar Leptospira spp. en pacientes de zonas endémicas. Este subregistro podría ser una situación común en todo el país.

Palabras clave: Leptospira/diagnóstico; cultivo de sangre; pruebas de aglutinación; técnica del anticuerpo fluorescente indirecta.

Incidence and underreporting of leptospirosis comparing three diagnostic methods in the endemic region of Urabá, Colombia

Introduction: Leptospirosis represents a public health problem and is a significant cause of morbidity and mortality in the region of Urabá. However, its notification reveals diagnostic limitations.

Objective: To establish the incidence of leptospirosis in the municipalities of the so-called eje bananero in the Urabá region, to describe the magnitude of underreporting, and to propose guidelines for laboratory diagnosis by the public health network.

Materials and methods: Two leptospirosis information sources were used: The national official surveillance system and a cross-sectional study of 479 acute-phase patients from April, 2010, to May, 2012. The diagnosis was made using three different tests: Indirect immunofluorescence, microagglutination test, and blood cultures. The exhaustiveness percentage of each information source was calculatedusing thecapture and recapture test. Results: From the total number of cases, 58\% (278/479) were positive for leptospirosis at least by a test and $10.43 \%$ (29/278) of cases were positive by all three methods. The inclusion of a native strain in the microagglutination test panel increased the percentage of positivity by $15 \%$. The cumulative incidence rate was $66.5 / 100,000$ inhabitants and the case fatality ratio was $2.15 \%$. The underreporting rates of leptospirosis in the Urabá region were $27.8 \%$ in morbidity and $66.6 \%$ in mortality.

Conclusion: Under-registration of leptospirosis in the region highlights the necessity to use more than one diagnostic test to identify Leptospira in patients from endemic areas. Underregistration could be a common situation throughout the country.

Keywords: Leptospira/diagnosis; blood culture; agglutination tests; fluorescent antibody technique; indirect. 
La leptospirosis es una zoonosis bacteriana desatendida (1). El $73 \%$ de los reportes se originan en el trópico y, en Latinoamérica, se registran entre 2.400 y 36.000 casos, en tanto que los casos fatales llegan a los 1.600 (2), por lo que se lo reconoce como el agente patógeno humano más frecuente causante de epidemias (3). La transmisión de Leptospira spp. y la expresión clínica de la enfermedad, se relacionan con las condiciones del entorno ambiental y socioeconómico, así como con el desplazamiento rural, la ruptura de las barreras ecológicas naturales y la poca disponibilidad de recursos sanitarios en las zonas rurales y urbanas; asimismo, el cambio climático, las inundaciones y el contacto frecuente entre el humano y sus múltiples reservorios naturales, favorecen el incremento de la incidencia $(4,5)$

En Colombia, se ha reportado una incidencia de 10,2 casos por 100.000 habitantes (6-9). Su inclusión como una de las zoonosis de notificación obligatoria en el Sistema Nacional de Vigilancia en Salud Pública (Sivigila) data del $2007(10,11)$. En el 2015, se reportaron 634 casos confirmados por laboratorio en todo el país, de los cuales 167 (26,1\%) procedían del departamento de Antioquia y, de estos, el 47,3 \% (79/167) se registró en la región del Urabá antioqueño (12), la cual comprende 11 municipios (figura 1).

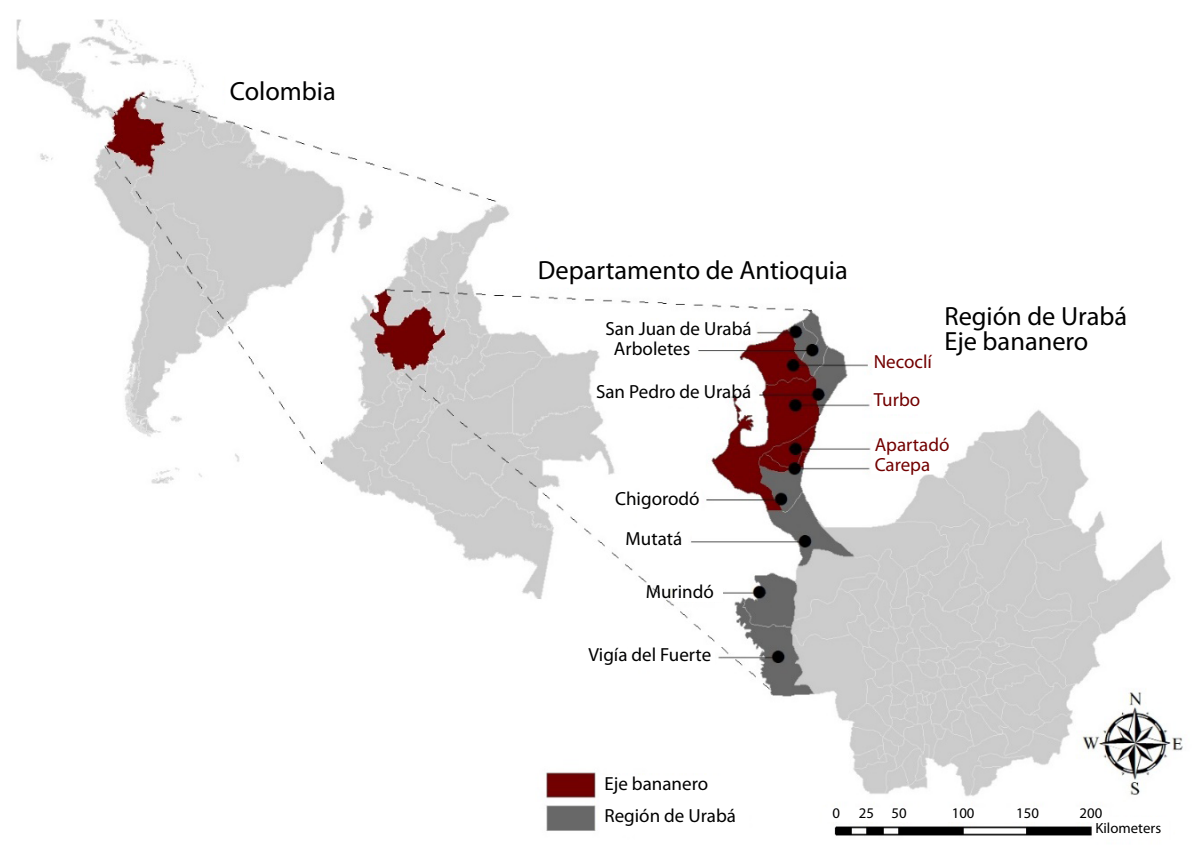

Figura 1. Área geográfica de estudio: región de Urabá, Antioquia, Colombia. Se resaltan en rojo los municipios del eje bananero (Chigorodó, Carepa, Apartadó y Turbo).

La región de Urabá es endémica para varias enfermedades tropicales que cursan como síndromes febriles agudos, algunos de ellos con manifestaciones hemorrágicas (9), como dengue, chikungunya, Zika, leptospirosis, rickettsiosis y malaria, cuyo diagnóstico por el laboratorio en los primeros niveles de atención solo está disponible para algunas de ellas (malaria y dengue), o para pacientes con sintomatología grave o de alerta (13).

La microaglutinación es la prueba de referencia para el diagnóstico de leptospirosis, aunque también pueden emplearse otros métodos, como la prueba ELISA (Enzyme-Linked ImmunoSorbent Assay), la inmunofluorescencia indirecta, las pruebas comerciales rápidas, los hemocultivos y los urocultivos 
en medios de cultivo comerciales. Varios autores recomiendan realizar dos pruebas diferentes $(14,15)$, y pruebas moleculares estandarizadas para el diagnóstico rutinario en las áreas endémicas (16).

El subregistro de la incidencia de la leptospirosis y de otras enfermedades febriles, tiene repercusiones a nivel local y nacional para el sistema de vigilancia epidemiológica, así como consecuencias económicas debidas a la hospitalización y a los días de incapacidad de los afectados. Se considera que el uso de las pruebas diagnósticas eficientes puede generar información que permita mejorar los criterios de evaluación de la vigilancia de la leptospirosis, especialmente, en los indicadores de diagnóstico y de tratamiento oportuno.

El objetivo del presente estudio fue establecer la incidencia de leptospirosis en cuatro municipios (Chigorodó, Carepa, Apartadó y Turbo) de los once del llamado 'eje bananero' de la región de Urabá, documentar las deficiencias en la notificación y proponer orientaciones para el diagnóstico por laboratorio en la red de salud pública.

\section{Materiales y métodos}

Se llevó a cabo un estudio transversal anidado en un proyecto de investigación sobre los factores epidemiológicos asociados con la gravedad de la leptospirosis en la región de Urabá. Se incluyeron 479 pacientes con síndrome febril agudo en cuatro municipios de la región, entre abril del 2010 y mayo del 2012. El diagnóstico se hizo mediante la detección de anticuerpos IgG e lgM en pruebas pareadas con inmunofluorescencia indirecta, técnica estandarizada en el Instituto Colombiano de Medicina Tropical, ICMT (15), microaglutinación y aislamiento de Leptospira spp. a partir del hemocultivo en medio de Fletcher y de Ellinghausen-McCullough-Johnson-Harris.

En la prueba de microaglutinación, se consideró positivo un título de 1:200 y se tuvieron en cuenta, además, los pacientes con seroconversión en la fase convaleciente. El panel serológico incluyó 12 serogrupos de cinco especies de referencia de Leptospira patógena y una cepa local: $L$. interrogans serogrupos Icterohaemorrhagiae, Canicola, Sejroe, Pomona y Australis; L. noguchii serogrupo Panama; L. kirschneri serogrupos Cynopteri y Grippotyphosa; L. borgpetersenii serogrupos Ballum y Tarassovi, y $L$. santarosai serogrupos Shermani y Autumnalis (cepa nativa aislada en el municipio de Apartadó). Hubo muestras en las que se evidenció la aglutinación con más de un serogrupo de Leptospira (17).

Se construyeron los canales endémicos de leptospirosis para el departamento de Antioquia, para la región de Urabá y para el eje bananero utilizando los casos diagnosticados por detección de anticuerpos lgM mediante ELISA reportados al Sivigila entre el 2008 y el 2015 (18).

Se obtuvieron medidas de frecuencia y se hizo la prueba de ji al cuadrado para determinar la relación entre las variables sociodemográficas y la positividad para leptospirosis; además, se calculó la diferencia de proporciones para la comparación de los resultados de las pruebas diagnósticas que incluyeron la cepa local en la microaglutinación.

Estos análisis se hicieron con el programa SPSS 21.0 ${ }^{\mathrm{TM}}$ (IBM, Armonk, NY) (bajo licencia de la Universidad CES), teniendo en cuenta un nivel de confianza del $95 \%$ y valores de p menores de 0,05 para evaluar la significación estadística. 
Las proporciones de subregistro se calcularon según la ecuación:

$$
\text { Subregistro }=100-\left(\frac{\text { Casos registrados por el Sivigila }}{\text { Total de casos incluidos en el estudio }}\right) \times 100
$$

Los pacientes en el estudio se captaron mediante vigilancia activa y aquellos registrados en el sistema oficial (Sivigila) se habían captado en la vigilancia pasiva a partir de los reportes de las entidades de salud de cada municipio del eje bananero.

Las tasas de incidencia por municipio y por grupo de edad se ajustaron directamente. El subregistro se cuantificó mediante el método de captura y recaptura (19), haciendo uso de los casos registrados en el Sivigila y los detectados en el estudio. Los dos procedimientos se hicieron con el programa Epidat 3.1 (Dirección Xeral de Saúde Pública, Xunta de Galicia, España).

\section{Resultados}

Durante el período de estudio, se captaron 479 pacientes con síndrome febril agudo en los cuatro municipios del eje bananero y se confirmó el diagnóstico de leptospirosis en el 58 \% (278/479) de los casos.

En la figura 2, se presenta el porcentaje de positividad según el método utilizado. Se observó que, con la prueba de inmunofluorescencia indirecta, hubo una mayor frecuencia diagnóstica $(65,1 \%)(181 / 278)$ y que el $10,43 \%$ (29/278) de los casos fue positivo con los tres métodos.

La inclusión de Leptospira santarosai serogrupo Autumnalis cepa JET (cepa nativa) en el panel de serogrupos de la microaglutinación, aumentó en $15 \%$ el porcentaje de positividad con respecto al panel convencional $(p=0,012)$.

Los serogrupos con mayor proporción de positividad en la prueba de microaglutinación fueron Leptospira santarosai serogrupo Autumnalis

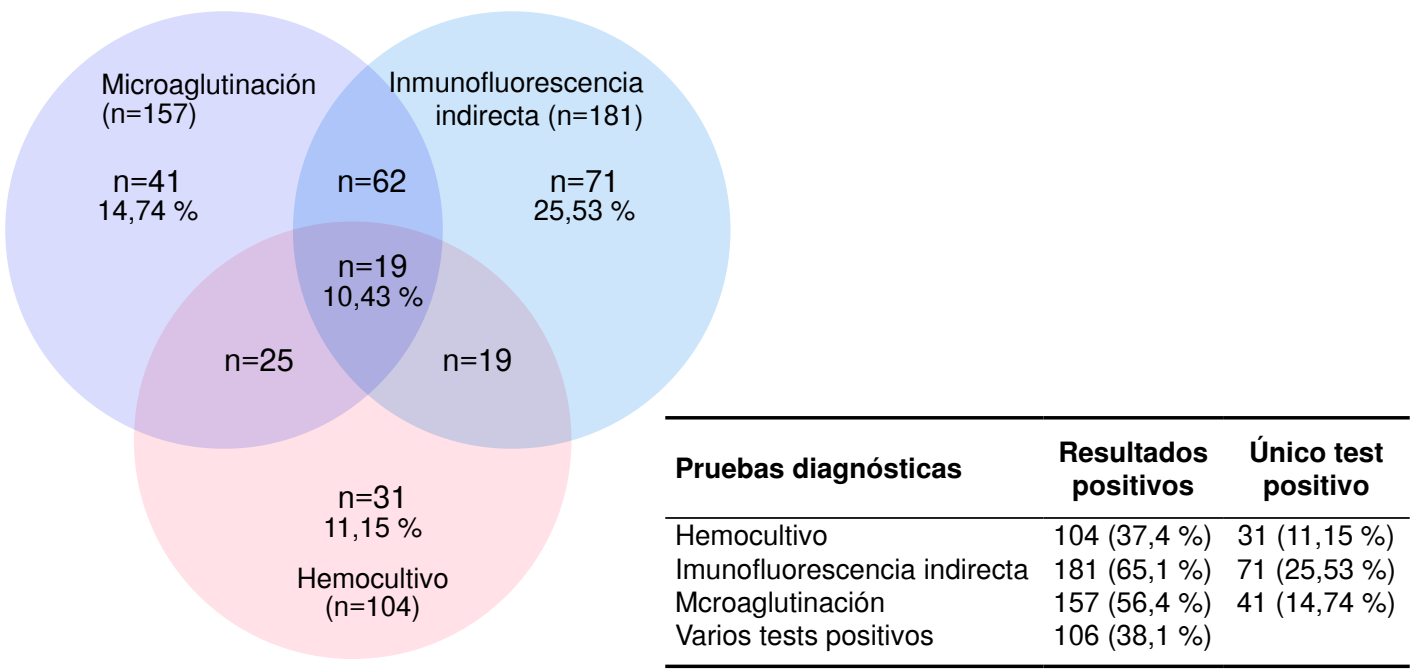

Figura 2. Casos positivos según la prueba utilizada para el diagnóstico de leptospirosis, Urabá, 2010-2012 
en 29/199 pacientes (14,57\%), Leptospira interrogans serogrupo Icterohaemorragiae en 23/199 (11,56 \%) y el serogrupo Sejroe en 20/199 (10,05\%). Otros serogrupos, como Australis, Tarassovi, Grippotyphosa, Shermani, Cynopteri, Canicola, Panama, Pomona y Barranquilla, registraron una frecuencia menor de $10 \%$, en tanto que el porcentaje de coaglutinación fue de $17,59 \%$ (35/199 casos).

En el cuadro 1 se presentan algunas características demográficas de los pacientes con leptospirosis incluidos en el estudio; se observó una mayor proporción de casos en hombres (186/278, 66,91\%), en habitantes de la zona urbana (198/278, 71,48\%), en los mestizos (130/278, 49,81\%) y en aquellos con edades entre los 20 y los 49 años (169/278, 61,23\%).

Cuadro 1. Características demográficas de la población incluida en el estudio (eje bananero, Urabá), 2010-2012

\begin{tabular}{|c|c|c|c|c|c|}
\hline & \multicolumn{2}{|c|}{ Pacientes positivos } & \multicolumn{2}{|c|}{ Pacientes negativos } & \multirow[t]{2}{*}{$\mathbf{p}$} \\
\hline & $\mathbf{n}$ & $(\%)$ & $\mathbf{n}$ & $(\%)$ & \\
\hline \multicolumn{6}{|l|}{ Sexo } \\
\hline Mujer & 92 & $(33,09)$ & 123 & $(61,2)$ & 0,117 \\
\hline Hombre & 186 & $(66,91)$ & 78 & $(38,8)$ & \\
\hline \multicolumn{6}{|l|}{ Zona de vivienda* } \\
\hline Urbana & 198 & $(71,48)$ & 143 & $(71,1)$ & 0,399 \\
\hline Rural & 79 & $(28,52)$ & 55 & $(27,4)$ & \\
\hline \multicolumn{6}{|l|}{ Grupo étnico } \\
\hline Blanco & 87 & $(33,33)$ & 169 & $(37,31)$ & 0,591 \\
\hline Mestizo & 130 & $(49,81)$ & 208 & $(45,92)$ & \\
\hline Negro & 40 & $(15,33)$ & 66 & $(14,57)$ & \\
\hline Indígena & 4 & $(1,53)$ & 4 & $(0,88)$ & \\
\hline \multicolumn{6}{|l|}{ Edad (años) ${ }^{*}$} \\
\hline $5-19$ & 87 & $(31,52)$ & 59 & $(24,18)$ & 0,000 \\
\hline $20-49$ & 169 & $(61,23)$ & 107 & $(43,85)$ & \\
\hline$>50$ & 20 & $(7,25)$ & 78 & $(31,97)$ & \\
\hline \multicolumn{6}{|l|}{ Ocupación* } \\
\hline Sector agropecuario & 18 & $(6,27)$ & 10 & $(4,98)$ & 0,000 \\
\hline Sector bananero & 22 & $(7,67)$ & 14 & $(6,97)$ & \\
\hline Militar & 28 & $(9,76)$ & 15 & $(7,46)$ & \\
\hline Ama de casa & 35 & $(12,20)$ & 39 & $(19,40)$ & \\
\hline Menor/estudiante & 84 & $(29,27)$ & 57 & $(28,36)$ & \\
\hline Otras & 100 & $(34,84)$ & 66 & $(32,84)$ & \\
\hline
\end{tabular}

* Variables con valores perdidos, por zona de vivienda: un caso $(0,35 \%)$; por edad: dos casos $(0,7 \%)$; por grupo étnico: 17 casos $(6,1 \%)$; por ocupación: 18 casos $(6,4 \%)$

No hubo asociación estadísticamente significativa entre la procedencia y la raza con respecto a la positividad ( $p=0,399$ y 0,591 , respectivamente) (cuadro 1 ).

En el cuadro 2 se presentan la distribución de los casos por año y por municipio, y las tasas de incidencia, crudas y ajustadas por edad, durante el período de estudio. Los 278 casos de leptospirosis diagnosticados suponen una incidencia acumulada total de 264 casos por 100.000 habitantes en los cuatro municipios del eje bananero, en tanto que los 145 casos registrados en el 2010 suponen una incidencia acumulada de 35,43/100.000 habitantes.

Para determinar el subregistro, se optó por trabajar con los datos del 2010 porque se ajustaban más a la realidad del proceso de vigilancia epidemiológica de los municipios del eje bananero antes del estudio. Para los años siguientes, se hizo el ajuste de la notificación al Sivigila con los casos que se diagnosticaron en el marco del presente estudio. 
Cuadro 2. Distribución de los casos de leptospirosis incluidos en el estudio y registro de incidencia por municipios del eje bananero, Urabá, 2010-2012

\begin{tabular}{|c|c|c|c|c|c|c|c|c|c|c|}
\hline & \multicolumn{2}{|c|}{ Apartadó } & \multicolumn{2}{|c|}{ Carepa } & \multicolumn{2}{|c|}{ Chigorodó } & \multicolumn{2}{|c|}{ Turbo } & \multicolumn{2}{|c|}{$\begin{array}{c}\text { Total de } \\
\text { casos por año }\end{array}$} \\
\hline & $\mathbf{n}$ & $(\%)$ & $\mathbf{n}$ & $(\%)$ & $\mathbf{n}$ & $(\%)$ & $\mathbf{n}$ & $(\%)$ & $\mathbf{n}$ & (\%) \\
\hline 2010 & 69 & $(45,7)$ & 8 & $(25,8)$ & 9 & $(69,2)$ & 59 & $(71,1)$ & 145 & $(52,2)$ \\
\hline 2011 & 70 & $(46,4)$ & 17 & $(54,8)$ & 3 & $(23,1)$ & 21 & $(25,3)$ & 111 & $(39,9)$ \\
\hline 2012 & 12 & $(7,9)$ & 6 & $(19,4)$ & 1 & $(7,7)$ & 3 & $(3,6)$ & 22 & $(7,9)$ \\
\hline $\begin{array}{l}\text { Total de casos por } \\
\text { municipio }\end{array}$ & 151 & 100 & 31 & 100 & 13 & 100 & 83 & 100 & 278 & 100 \\
\hline $\begin{array}{l}\text { Incidencia cruda } \\
\text { por } 100.000 \\
\text { habitantes }\end{array}$ & \multicolumn{2}{|c|}{95,5} & \multicolumn{2}{|c|}{59,5} & \multicolumn{2}{|c|}{20,3} & \multicolumn{2}{|c|}{57,8} & & \\
\hline $\begin{array}{l}\text { Incidencia ajustada } \\
\text { por edad por } \\
100.000 \text { habitantes }\end{array}$ & \multicolumn{2}{|c|}{90} & \multicolumn{2}{|c|}{60} & \multicolumn{2}{|c|}{20} & \multicolumn{2}{|c|}{50} & & \\
\hline
\end{tabular}

En la figura 3, se presentan los canales endémicos basados en la información oficial (Sivigila) para el país, el departamento, la región de Urabá y los municipios del eje bananero. Se puede observar la endemia de la leptospirosis en todas las zonas, con una tendencia hiperendémica a partir del periodo epidemiológico ocho antes del inicio de la segunda temporada anual de lluvias, tendencia que se mantuvo hasta finalizar el año. Los cuatro municipios del eje bananero aportaron el 88,67 \% de los casos de la región de Urabá.
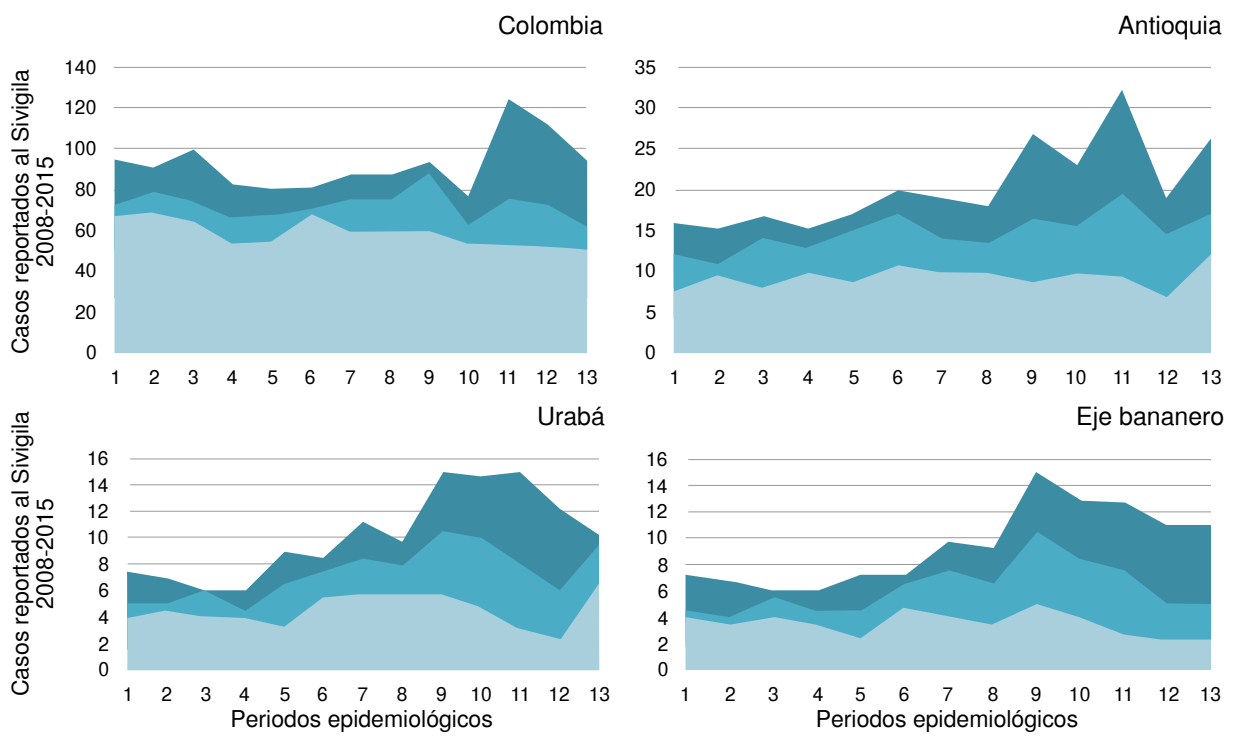

Figura 3. Canal endémico de leptospirosis según los casos reportados por el Sivigila (20082015) en Colombia, en Antioquia, en la región de Urabá y en el eje bananero (municipios de Apartadó, Carepa, Chigorodó y Turbo). Se observa endemia en todas las zonas, con una tendencia hiperendémica a partir del periodo epidemiológico ocho y hasta finalizar el año. Los municipios del eje bananero aportaron el 88,67 \% de los casos de la región de Urabá.

En la figura 4 se presenta la distribución proporcional de los casos entre el 2008 y el 2015 según la notificación al Sivigila en los cuatro municipios de estudio, en los demás municipios de la región de Urabá y en los municipios restantes del departamento de Antioquia. Se observó una tendencia al aumento en el reporte de casos de los municipios de la región de Urabá, que se mantuvo constante a partir del 2010. El eje bananero reportó proporciones por encima del $40 \%$ a partir de ese año. 


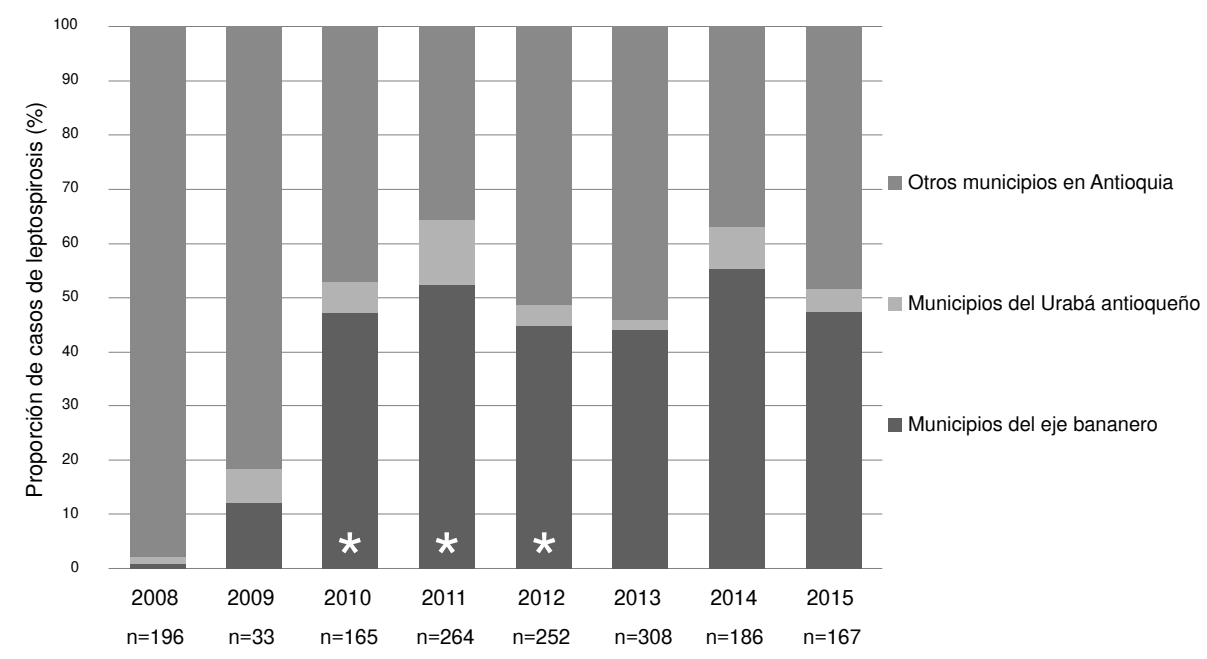

* Periodo del estudio

Figura 4. Casos de leptospirosis por año en Antioquia reportados en el Sivigila (2008-2015) discriminados según la procedencia: municipios del eje bananero, Urabá antioqueño y resto del departamento de Antioquia. Se observó una tendencia al aumento en el reporte de casos de los municipios de la región de Urabá, con reportes constantes a partir del 2010. A partir de esta fecha, en el eje bananero se reportaron proporciones por encima del $40 \%$.

Según las estimaciones hechas mediante el método de captura y recaptura, se debieron haber notificado al Sivigila 230 casos de leptospirosis en los municipios del eje bananero durante el 2010 (IC ${ }_{95 \%}$ 191-269), y la incidencia estimada mediante este mismo método debió haber oscilado entre 46,66 y 65,72 por 100.000 habitantes.

De los 126 pacientes notificados en el estudio, 56 también fueron reportados al Sivigila y 70 no, en tanto que el total de los que no se notificaron al Sivigila fue de 104. Con esta información y con el método propuesto, se calculó la exhaustividad en la captación de pacientes con ambas fuentes de información (el presente estudio y la información del Sivigila), porcentaje equivalente al $72,12 \%$, lo que implica que, incluso tomando los datos de las dos fuentes, el $27,88 \%$ de casos de leptospirosis en el 2010 no fueron diagnosticados. En la figura 5 se presenta el porcentaje de casos registrados en el Sivigila, discriminado por municipio y por año.

El total de casos notificados al Sivigila por los cuatro municipios durante el período de estudio, fue de 69, mientras que el estudio captó 278 casos en forma activa, es decir que el subregistro oficial fue de $75,18 \%$. Durante el 2012, no se reportó ningún caso al Sivigila en Chigorodó y solo un paciente de este municipio se incluyó en el estudio. En la figura 5, se presenta el subregistro de los municipios de estudio, Apartadó, Carepa, Chigorodó y Turbo, en cada uno de los años. La proporción de casos que no se registraron fue de $75,86 \%$ en el 2010 , de $76,58 \%$ en el 2011 y de $63,64 \%$ en el 2012 .

Durante el período de estudio, se notificaron dos casos fatales al Sivigila, uno de ellos procedente del municipio de Apartadó y otro del municipio de Turbo, aunque en el estudio se documentaron seis casos fatales, lo cual indica que hubo cuatro casos fatales que no se notificaron al Sivigila, para una proporción de letalidad por leptospirosis del 2,15\% en los municipios del eje bananero, una tasa de mortalidad de 1,27 por 100.000 habitantes y un subregistro de la letalidad del 66,6\% (4/6). 


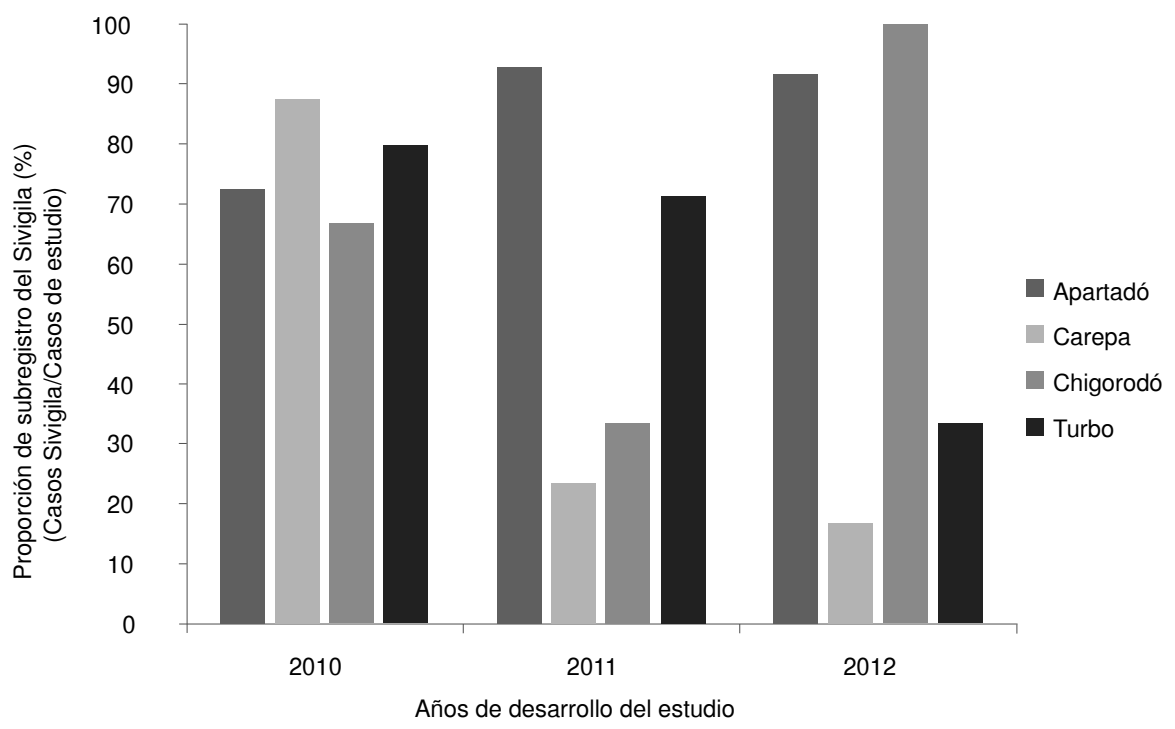

Figura 5. Proporción de los casos no registrados en el Sivigila (2010-2012) según los reportes del presente estudio discriminados por los municipios del eje bananero

\section{Discusión}

Los resultados del presente estudio evidenciaron 278 (58,03\%) pacientes positivos para leptospirosis en, al menos, una de las pruebas utilizadas: hemocultivo, detección de anticuerpos por inmunofluorescencia indirecta o microaglutinación, en la fase aguda o la fase convaleciente de una cohorte de 479 pacientes febriles captados en los municipios del eje bananero. Este porcentaje de positividad en los pacientes febriles superó lo descrito en otros estudios de caracterización de la etiología del síndrome febril agudo en Colombia. Arroyave, et al., encontraron $14,1 \%$ de positividad de leptospirosis mediante inmunofluorescencia indirecta en pacientes de tres municipios de Urabá (20). En el departamento del Quindío se reportaron porcentajes de positividad mediante microaglutinación de 6,8\% en el 2016 (17) y de $5,7 \%$ y $4,7 \%$ en el 2005 y el 2006 , respectivamente (6). Estas diferencias probablemente se explican por el uso de un único método diagnóstico con diferentes sensibilidades y especificidades en cada estudio.

El diagnóstico de la leptospirosis es complejo; una única prueba de laboratorio no lo garantiza por sí misma, y se recomienda que, en los pacientes con sospecha de tenerla, se utilicen, por lo menos, dos pruebas de laboratorio, preferentemente, una serológica acompañada de una microbiológica o molecular (4).

Según la Organización Mundial de la Salud (OMS) y los protocolos del Instituto Nacional de Salud de Colombia, la prueba estándar para el diagnóstico de la enfermedad es la detección de anticuerpos mediante microaglutinación $(21,22)$. Sin embargo, el diagnóstico estandarizado en la red nacional de laboratorios de salud pública es la detección de anticuerpos IgM mediante ELISA, lo que restringe la determinación exclusivamente a la fase aguda, excluyendo la fase de convalecencia.

En cuanto a la microaglutinación, se puede afirmar que su sensibilidad depende del panel de serovares disponible en el laboratorio, lo que puede limitar el diagnóstico (21-23) cuando no se incluyen cepas nativas de las áreas endémicas (24). Esto se hizo evidente en el presente estudio al incluir 
la cepa nativa JET, aislada de un paciente procedente del municipio de Apartadó, en el panel de diagnóstico de la microaglutinación (25), ya que ello mejoró la captación de pacientes positivos en $15 \%$ con respecto al panel convencional, diferencia estadísticamente significativa.

El $11,1 \%(31 / 278)$ de los pacientes del estudio con resultado negativo en la inmunofluorescencia indirecta y la microaglutinación, se diagnosticó únicamente por cultivo. Aunque el cultivo es un método imperfecto, representa una posibilidad diagnóstica en aquellos casos en que no desarrollan anticuerpos contra Leptospira spp. En este estudio se hizo el seguimiento de los hemocultivos durante nueve meses, seis meses más de los recomendados por la OMS (21), lo cual pudo contribuir al notable rendimiento alcanzado pues, en un poco más de la tercera parte de los casos (37,4 \%; 104/278), el diagnóstico se obtuvo por este medio.

Desde el punto de vista epidemiológico, la información que aporta el cultivo es muy importante, aunque no es útil como apoyo para definir el tratamiento. Dadas las limitaciones del cultivo, las pruebas moleculares basadas en la reacción en cadena de la polimerasa (PCR) constituyen una alternativa diagnóstica (26). Sin embargo, cuando no se cuenta con la infraestructura necesaria para el diagnóstico molecular en los primeros niveles de atención (instalaciones especiales, capacitación y experiencia del personal, y nivel de seguridad, entre otros), el diagnóstico puede hacerse mediante dos pruebas simples y muy sensibles: la de campo oscuro de la orina y una prueba rápida validada con serovares nativos de las zonas endémicas, para garantizar un tratamiento más específico. En el diagnóstico de pacientes de áreas endémicas para leptospirosis, se considera válida la combinación de las pruebas básicas de laboratorio con los criterios clínicos definidos (como los descritos y modificados por Faine en 1999), especialmente, en lugares con pocos recursos para implementar otro tipo de métodos diagnósticos $(27,28)$.

El contexto de la epidemiología dinámica de la enfermedad determina que las poblaciones expuestas a los factores de riesgo dejaron de ser exclusivamente del área rural y de las ocupaciones de riesgo (trabajadores del agro, ganaderos, veterinarios, etc.), como lo demuestran los resultados del presente estudio, en el que la mayoría de los casos correspondió a personas procedentes del área urbana (198/278 71,48\%), hallazgos similares a los reportados en la literatura médica $(2,29)$. En este mismo sentido, en un estudio de seroprevalencia de la leptospirosis en la región de Urabá publicado en el 2007, se reportó un perfil sociodemográfico similar (30), lo que demuestra que otros factores ambientales (lluvias, inundaciones, mascotas, animales sinantrópicos, prácticas deportivas, etc.) han contribuido a modificar el comportamiento epidemiológico y de los factores de riesgo de la enfermedad, especialmente, en los sectores urbanos deprimidos con deficientes condiciones higiénicas y sanitarias (31).

Este cambio epidemiológico y el subregistro de los casos de leptospirosis tienen implicaciones clínicas, pero también subestiman la carga de la enfermedad y sus implicaciones económicas. En el 2015, el 79,6\% de los pacientes diagnosticados con leptospirosis en Colombia requirió manejo hospitalario, y el $53 \%$ estaba asegurado en el régimen subsidiado por el Estado (12); el tiempo de incapacidad fue de cerca de 12 días, con la consecuente ausencia del lugar de trabajo, lo cual se traduce no solo en un costo adicional para el empleador, sino también para la economía de la región, que depende del sector productivo primario y de su mano de obra. 
Los cuatro municipios incluidos del eje bananero, (Turbo, Apartadó, Carepa y Chigorodó) representan el 6,74 \% de la población de Antioquia y notifican más del $40 \%$ del total de casos de leptospirosis del departamento. Los 145 casos incluidos en el estudio durante el 2010, representan una tasa de incidencia de 35,4 casos por 100.000 habitantes, lo que superó las tasas promedio de Antioquia (2,72 por 100.000 habitantes) y de Colombia (2,71 por 100.000 habitantes), estimadas oficialmente para ese mismo año, así como las de otras regiones en el país (eje cafetero, con 23,41 casos por 100.000 habitantes en el 2011) (32) y en Latinoamérica (Lima: 56,3 por 100.000 habitantes) (2).

En su estudio sobre la dinámica espacio-temporal de la leptospirosis humana y la relación con las anormalidades climáticas, Gutiérrez, et al., determinaron uno de los seis conglomerados con mayor número de casos de leptospirosis humana en Colombia, correspondiente a los municipios del eje bananero, y lo asociaron con la incidencia del comportamiento endémico y a una mayor descarga de lluvias debida, probablemente, al fenómeno de La Niña, entre otros aspectos (33).

En este mismo sentido, en los canales endémicos basados en la información del Sivigila, se evidenció una clara tendencia al aumento, principalmente en los municipios de la región de Urabá, a partir de los últimos cinco períodos epidemiológicos del año, época en la cual suelen aumentar las lluvias y, por ende, las aguas estancadas y servidas que aumentan la exposición y el riesgo de desarrollar la enfermedad. Esta observación está documentada por los mismos autores en su estudio sobre agrupaciones de leptospirosis humana en los municipios del eje bananero (33).

En Urabá hay un elevado registro de la enfermedad, lo que evidencia los esfuerzos continuos de búsqueda de síndromes febriles sugestivos de leptospirosis y el compromiso de los funcionarios del área de la salud con su notificación, lo cual se ha reflejado en su incremento a partir de la realización de este estudio, como se muestra en la figura 3. Probablemente, la búsqueda activa de los casos contribuyó al aumento de la incidencia en esta zona, aunque se sigue evidenciando un subregistro notable en los datos de vigilancia, lo cual se asociaría indirectamente con el hecho de no disponer de pruebas diagnósticas eficientes en la red de laboratorios de salud pública.

En la revisión sobre la vigilancia de la leptospirosis en las Américas entre 1996 y 2005, se evidenció la falta de datos sobre la enfermedad, lo cual refleja las deficiencias de los sistemas de vigilancia, la poca observancia de la notificación obligatoria, la debilidad del diagnóstico por el laboratorio y de la capacidad para recolectar los casos notificados, así como la urgencia de detectar tempranamente los de brotes, hacer un seguimiento preciso de los patrones regionales y establecer la carga de la enfermedad $(2,34)$.

Entre el 2010 y el primer semestre de 2012, no se registraron casos fatales por malaria y solo una muerte por dengue (35), en tanto que se documentaron seis casos fatales de leptospirosis: tres del municipio de Apartadó, dos de Turbo y uno de Chigorodó, lo cual indica una proporción de letalidad de 2,15\% en los municipios del eje bananero. Estos hallazgos reiteran el planteamiento de que la leptospirosis representa un grave problema de morbimortalidad en la región de Urabá que no se ve reflejado en el sistema de vigilancia.

Asimismo, se observó que la notificación de la letalidad de la enfermedad en el Sivigila no es la adecuada, pues se reportaron solo dos de los seis casos encontrados en el presente estudio. En Puerto Rico, se dejaron de notificar 20 casos de leptospirosis y cinco muertes por esta misma causa, entre el 2000 y 
el 2009, lo que indica que no se notificó entre el 60 y el $90 \%$ de la letalidad, datos comparables a los encontrados en el presente estudio (36).

El subregistro promedio del $72 \%$ de los casos y del $66 \%$ de los fallecidos en la región es considerablemente alto. Los 230 casos de leptospirosis que debieron haberse notificado al Sivigila suponen una incidencia estimada que debió haber oscilado entre 46,66 y 65,72 por 100.000 habitantes, tasa cinco veces mayor que la registrada en ese Sistema en el 2010 para los municipios del eje bananero, la cual fue de 11,365 por 100.000 habitantes. Un estudio de leptospirosis humana en Brasil, en el cual se empleó el método de captura y recaptura, reportó un subregistro 20 veces mayor que el estimado por las fuentes oficiales (37). Este subregistro debe alertar a las autoridades sanitarias para garantizar un mejor acceso al diagnóstico en la red de laboratorios de salud pública, ya que la detección de anticuerpos mediante ELISA, usualmente en fase aguda, es insuficiente por sí sola para dar cuenta de esta enfermedad endémica en los municipios del estudio.

Este panorama plantea la necesidad de que la red nacional de laboratorios de salud pública adopte, por lo menos, una prueba complementaria, preferentemente microbiológica, u otra prueba serológica para la vigilancia de leptospirosis, medida que contribuiría a mejorar el diagnóstico de la enfermedad y, en consecuencia, el perfil epidemiológico, al disminuir el subregistro actual. Además, las pruebas moleculares basadas en la PCR son una alternativa diagnóstica ya validada en Colombia (38).

En conclusión, es necesario disponer, por lo menos, de dos pruebas diagnósticas debido a la historia natural de la enfermedad, el comportamiento de la bacteria, el corto periodo de leptospiremia y el desarrollo retardado o la ausencia de anticuerpos en algunos pacientes $(24,39)$. Asimismo, el diagnóstico oportuno y preciso de la leptospirosis en los pacientes febriles de las zonas endémicas para otras enfermedades, como dengue, chikungunya y Zika, puede mejorar el pronóstico, pues permite un tratamiento adecuado y disminuir el subregistro de esta enfermedad en forma significativa.

\section{Referencias}

1. Chikeka I, Dumler JS. Neglected bacterial zoonoses. Clin Microbiol Infect. 2015;21:404-15. https://doi.org/10.1016/j.cmi.2015.04.022

2. Costa F, Hagan JE, Calcagno J, Kane M, Torgerson P, Martinez-Silveira MS, et al. Global morbidity and mortality of leptospirosis: A systematic review. PLoS Negl Trop Dis. 2015;9:119. https://doi.org/10.1371/journal.pntd.0003898

3. Dolhnikoff M, Mauad T, Bethlem EP, Carvalho CR. Leptospiral pneumonias. Curr Opin Pulm Med. 2007;13:230-5. https://doi.org/10.1097\%2FMCP.0b013e3280f9df74

4. Bharti AR, Nally JE, Ricaldi JN, Matthias MA, Diaz MM, Lovett MA, et al. Leptospirosis: A zoonotic disease of global importance. Lancet Infect Dis. 2003;3:757-71. https://doi.org/10.1016/S1473-3099(03)00830-2

5. Adler B. Leptospira and leptospirosis. Berlín: Springer Berlin Heidelberg; 2015. https://doi.org/10.1007/978-3-662-45059-8

6. Sánchez-Vallejo G, Gómez-Marín J, Quintero-Álvarez L, Castaño-Cotrina M. Características clínicas y epidemiológicas de la leptospirosis en el departamento del Quindío, 2005-2006. Infectio. 2008;12:325-31.

7. Padmanabha H, Hidalgo M, Valbuena G, Castañeda E, Galeano A, Puerta H, et al. Geographic variation in risk factors for SFG rickettsial and leptospiral exposure in Colombia. Vector-Borne Zoonotic Dis. 2009;9:483-90. https://doi.org/10.1089/vbz.2008.0092

8. Bravo C, Restrepo R, Pérez G. Leptospirosis in Antioquia. Preliminary communication. Antioquia Med. 1966;16:534-4. 
9. Epstein PR, Calix-Peña O, Blanco-Racedo J. Climate and disease in Colombia. Lancet. 1995;346:1243-4. https://thelancet.com/journals/lancet/article/PIIS0140-6736(95)91856-6/fulltext

10. Bello S, Rodríguez M, Paredes A, Mendivelson F, Walteros D, Rodríguez F, et al. Comportamiento de la vigilancia epidemiológica de la leptospirosis humana en Colombia, 2007-2011. Biomédica. 2013;33:153-60. https://doi.org/10.7705/biomedica.v33i0.1608

11. Ferro BE, Rodríguez AL, Pérez M, Travi BL. Seroprevalencia de infección por Leptospira en habitantes de barrios periféricos de Cali. Biomédica. 2006;26:250-7. https://doi.org/10.7705/biomedica.v26i2.1414

12. Instituto Nacional de Salud. Boletín Epidemiológico. Semana 52 de 2015. Sivigila. Fecha de consulta; 24 de abril del 2019, https://www.ins.gov.co/buscador-eventos/ BoletinEpidemiologico/2015\%20Boletin\%20epidemiologico\%20Semana\%2052.pdf

13. Ministerio de la Protección Social, Instituto Nacional de Salud, Organización Panamericana de la Salud. Guía para la atención clínica integral del paciente con dengue. Bogotá: Ministerio de la Protección Social, Instituto Nacional de Salud, Organización Panamericana de la Salud; 2010.

14. Yuszniahyati Y, Kenneth FR, Daisy Vanitha J. Leptospirosis: Recent incidents and available diagnostics - a review. Med J Malaysia. 2015;70:351-5.

15. Agudelo-Flórez P, Restrepo M, Lotero MA. Evaluación de la prueba de inmunofluorescencia indirecta para el diagnóstico de leptospirosis humana. Biomédica. 2006;26:216-23. https://doi.org/10.7705/biomedica.v26i2.1411

16. Agudelo-Flórez P, Durango H, Aranzazu D, Rodas JD, Travi B. Genotipificación y evaluación de la dinámica de infección de un aislamiento colombiano de Leptospira santarosai en el modelo experimental hámster. Biomédica. 2014;34:460-72. https://doi.org/10.7705/biomedica.v34i3.1703

17. Rodríguez-Salazar CA, Recalde-Reyes DP, González MM, Padilla-Sanabria L, QuinteroÁlvarez L, Gallego-Gómez JC, et al. Manifestaciones clínicas y hallazgos de laboratorio de una serie de casos febriles agudos con diagnóstico presuntivo de infección por el virus dengue. Quindío (Colombia). Infectio. 2016;20:84-92. https://doi.org/10.1016/j.infect.2015.08.003

18. Instituto Nacional de Salud. Protocolo de vigilancia en salud pública. Leptospirosis Cod. 455. Fecha de consulta: 24 de abril de 2015. Disponible en: https://www.ins.gov.co/buscador-eventos/Lineamientos/PRO\%20Leptospirosis.pdf

19. Xunta de Galicia, Consellería de Sanidade e o Servizo Galego de Saúde. Epidat 3.1. Fecha de consulta: 27 de abril de 2018. Disponible en:

https://www.xunta.gal/tema/c/Saude_asistencia sanitaria e servizos sociais

20. Arroyave E, Londoño AF, Quintero JC, Agudelo-Flórez P, Arboleda M, Díaz FJ, et al. Etiología y caracterización epidemiológica del síndrome febril no malárico en tres municipios del Urabá antioqueño, Colombia. Biomédica. 2013;33:99-107. https://doi.org/10.7705/biomedica.v33i0.734

21. Organización Panamericana de la Salud, Organización Mundial de la Salud, International Leptospirosis Society. Leptospirosis humana: Guía para el diagnóstico, vigilancia y control. Fecha de consulta: 7 de febrero de 2015. Disponible en: http://www.med.monash.edu.au/microbiology/staff/adler/guia-esp.pdf

22. Guerra MA. Leptospirosis: Public health perspectives. Biol J Int Assoc Biol Stand. 2013;41:295-7. https://doi.org/10.1016/j.biologicals.2013.06.010

23. Budihal SV, Perwez K. Leptospirosis diagnosis: Competency of various laboratory tests. J Clin Diagn Res. 2014;8:199-202. https://doi.org/10.7860/JCDR/2014/6593.3950

24. Picardeau M. Diagnosis and epidemiology of leptospirosis. Med Mal Infect. 2013;43:1-9. https://doi.org/10.1016/j.medmal.2012.11.005

25. Peláez-Sánchez RG, López JÁ, Pereira MM, Arboleda-Naranjo M, Agudelo-Flórez P. Genetic diversity of Leptospira in northwestern Colombia: First report of Leptospira santarosai as a recognized leptospirosis agent. Mem Inst Oswaldo Cruz. 2016;111:737-44. https://doi.org/10.1590/0074-02760160245

26. Moreno N, Agudelo-Flórez P. Aplicación de las pruebas de PCR convencional simple y múltiple para la identificación de aislamientos de Leptospira spp. en Colombia. Rev Peru Med Exp Salud Pública. 2010;27:548-56.

27. Bandara K, Weerasekera MM, Gunasekara C, Ranasinghe N, Marasinghe C, Neluka F. Utility of modified Faine's criteria in diagnosis of leptospirosis. BMC Infect Dis. 2016;16:1-7. https://doi.org/10.1186/s12879-016-1791-9 
28. Haake D. Leptospirosis in humans. Curr Top Microbiol Immunol. 2015;387:65-97. https://link.springer.com/chapter/10.1007\%2F978-3-662-45059-8 5

29. Sarkar U, Nascimento SF, Barbosa R, Martins R, Nuevo H, Kalofonos I, et al. Populationbased case-control investigation of risk factors for leptospirosis during an urban epidemic. Am J Trop Med Hyg. 2002;66:605-10. https://doi.org/10.4269/ajtmh.2002.66.605

30. Agudelo-Flórez P, Restrepo-Jaramillo BN, Arboleda-Naranjo M. Situación de la leptospirosis en el Urabá antioqueño colombiano: estudio seroepidemiológico y factores de riesgo en población general urbana. Cad Saúde Pública. 2007;23:2094-102. https://doi.org/10.1590/S0102-311X2007000900017

31. Hagan JE, Moraga P, Costa F, Capian N, Ribeiro GS, Wunder EA, et al. Spatiotemporal determinants of urban leptospirosis transmission: Four-year prospective cohort study of slum residents in Brazil. PLoS Negl Trop Dis. 2016;10:1-16. https://doi.org/10.1371/journal.pntd.0004275

32. García-Ramírez LM, Giraldo-Pulgarín JY, Agudelo-Marín N, Holguín-Rivera YA, GómezSierra S, Ortiz-Revelo PV, et al. Geographical and occupational aspects of leptospirosis in the coffee-triangle region of Colombia, 2007-2011. Recent Pat Antiinfect Drug Discov. 2015;10:42-50. https://doi.org/10.2174/1574891X10666150410130425

33. Gutiérrez JD, Martínez-Vega RA. Spatiotemporal dynamics of human leptospirosis and its relationship with rainfall anomalies in Colombia. Trans R Soc Trop Med Hyg. 2018;112:11523. https://doi.org/10.1093/trstmh/try032

34. Costa F, Martínez-Silveira MS, Hagan JE, Hartskeerl RA, Dos Reis MG, Ko Al. Surveillance for leptospirosis in the Americas, 1996-2005: A review of data from ministries of health. Pan Am J Public Health. 2012;32:169-77. https://doi.org/10.1590/S1020-49892012000900001

35. Secretaría Seccional de Salud y Protección Social de Antioquia. Mortalidad general por subregión/municipio, 2015-17. Fecha de consulta: 24 de abril de 2019. Disponible en: http://dssa.gov.co/index.php/estadisticas/mortalidad/item/83-mortalidad-enfermedadestransmitidas-por-vectores-por-subregion-municipio-2005-2017

36. Centers for Disease Control and Prevention. Notes from the field: Investigation of leptospirosis underreporting - Puerto Rico, 2010. MMWR Morb Mortal Wkly Rep. 2012;61:421.

37. Brum L, Kupek E. Record linkage and capture-recapture estimates for underreporting of human leptospirosis in a Brazilian health district. Braz J Infect Dis. 2005;9:515-20. https://doi.org/10.1590/S1413-86702005000600011

38. Agudelo-Flórez P, Moreno N, Restrepo M. Diagnóstico de leptospirosis de muestras de sangre y cultivo por observación en microscopio de campo oscuro. Biomédica. 2008;28:7-9. https://doi.org/10.7705/biomedica.v28i1.103

39. Jaiswal N, Chandrasekaran S, Padmavathy B. Dark field microscopy an important conventional technique for the early diagnosis of leptospirosis. Int J Curr Microbiol App Sci. 2015;4:718-22. 